\title{
Analysis and Calculation of Miller Capacitor in Amplifier for 8 Bits Pipeline ADC
}

\author{
Liu Ying $^{1}$, Xie Chi $^{2}$ and Zhang Yourun ${ }^{3}$ \\ ${ }^{1}$ School of Energy Science and Engineering, University of Electronic Science and Technology of \\ China, Chengdu, 611731, China \\ 2 Department of Mechanical Engineering, Jincheng College of Sichuan University, Chengdu, \\ 611731, China \\ ${ }^{3}$ School of Microelectronics and Solid-State Electronics, University of Electronic Science and \\ Technology of China, Chengdu, 611731, China
}

Keywords: pipeline ADC, Miller capacitor, amplifier, CMOS technology

Abstract. This design pays much attention on analyzing and calculating Miller capacitor in operational amplifier to optimist OP (Operational Amplifier). The design for a 8-bit high speed, low power pipeline ADC is based on standard 0.18um CMOS technology, with 3.3 power supply. The results show that the dc gain of the operational amplifier is $96 \mathrm{~dB}$, the gain-bandwidth is $167 \mathrm{MHZ}$, and the ADC can achieve 8bits resolution and 10M samples/s.

\section{Introduction}

System-on-chip (SOC) requires the integration of analog circuits and digital circuits on a signal chip. Recently, the applications for ADCs have expanded widely as many electronic systems that used to be entirely analog have been implemented using digital electronics [1]. It is very critical to design a high performance A/D converter, which is based on standard CMOS technology. Recently, the applications for ADCs have expanded widely as many electronic systems [2,3]. The pipeline ADC can achieve both high accuracy (6-14 bit), and high conversion speed (5MHZ-200MHZ) [4]. The pipelined ADC is serial work, but it is working in parallel from every step of the conversion [5]. So its total conversion rate depends on the maximum speed of a single-stage circuit, and the total conversion rate is no relation with the numbers of the stages. In this paper, we pay attention on analyzing and calculating Miller capacitor in operational amplifier to optimist the amplifier. So we design an 8-bit high speed, low power pipeline ADC based on the standard 0.18um CMOS technology.

\section{Principle of pipelined ADC}

In the high-speed and high precision ADC, the OP needs a high enough gain and gain bandwidth. In order to achieve the requirements, we used the fully differential CMOS operation amplifier and the gain improves technology. The OP is a two-stage CMOS operational amplifier, and it is showed in Fig.1. In Fig.1, it includes a differential input stage, a load capacitance $C_{L}$, and a stable transconductance bias circuit. Because the load capacitance $\mathrm{C}_{\mathrm{L}}$ is often several pf, the output drive level is no need. The CMOS two-stage operational amplifier has many advantages: high voltage gain, rail-to-rail output dynamic range, a large common-mode input range, a Miller compensation capacitor, etc.. But its main drawback is the second pole (non-dominant pole) is determined by the product the load capacitance and the second amplifier output impedance, which reduces the bandwidth of the op amp. Another drawback is the OP has the right-half plane zero which is caused by the compensation capacitors from the feed-forward pathway. But the polar can be eliminated by selecting the appropriate compensated resistor: linear region of a NMOS (M14). We can achieve the zero point moving by adjusting its width to length ration and gate-source voltage. Because the op is the differential input, so the dominant pole compensation is mainly influenced by the Miller compensation capacitor Cc. 


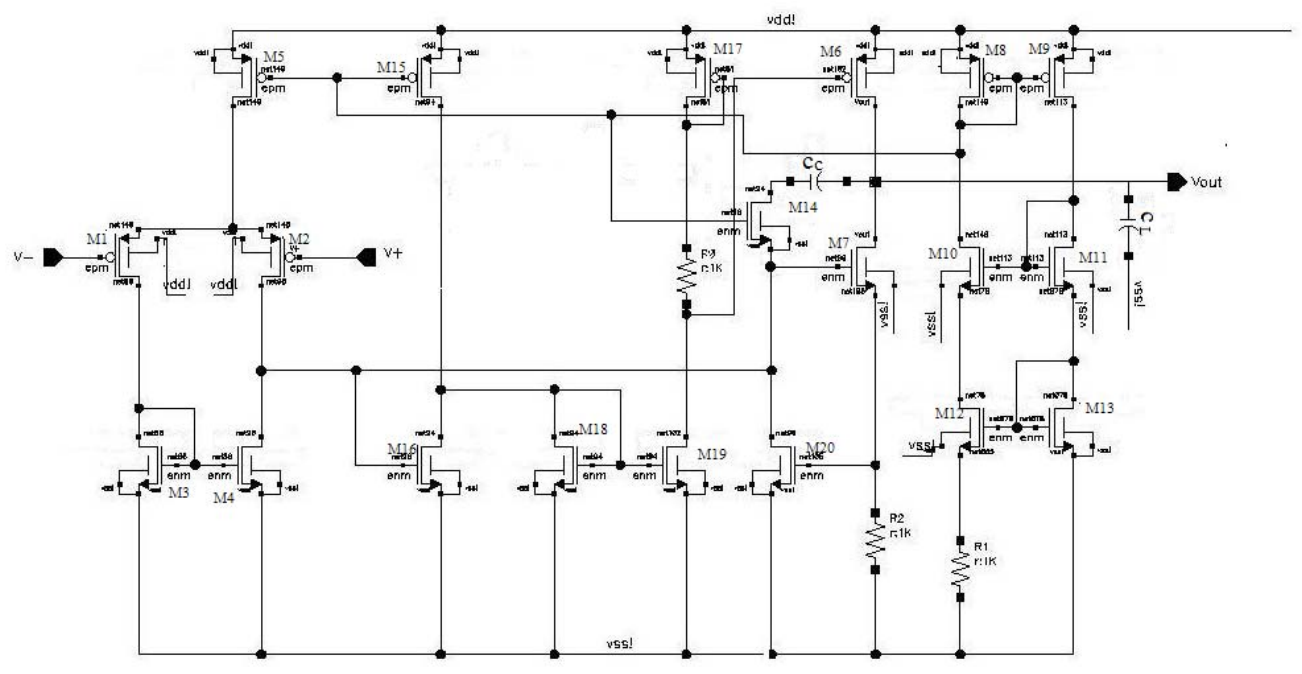

Fig.1 the structure of OP

The small signal model can simplify the calculation of the linear model, and it can be regarded as the ratio amount of the small changes in the large signal model parameters. $g_{m}$ is the channel admittance, and in the saturation region it is:

$$
g_{m}=\sqrt{\left(2 K^{\prime} W / L\right)\left|I_{D}\right|\left(1+\lambda v_{D S}\right)} \approx \sqrt{\left(2 K^{\prime} W / L\right)\left|I_{D}\right|}
$$

$g_{0}$ is the channel conductance for small signal

$$
g_{0}=\frac{I_{D} \lambda}{1+\lambda V_{D S}} \approx I_{D} \lambda
$$

$\mathrm{W}$ is the effective channel width, and $\mathrm{L}$ is the effective channel length, $\lambda$ is the modulation factor of the channel length $\left(\mathrm{V}^{-1}\right)$.The first pole is :

$$
w_{p 1} \approx \frac{g_{02}+g_{04}}{\left(\frac{g_{m 6}}{g_{06}+g_{07}}\right) C_{c}}
$$

Open loop gain is:

$$
A_{0}=\frac{g_{m 1}}{g_{02}+g_{04}} \cdot \frac{g_{m 6}}{g_{06}+g_{07}}
$$

Unity gain bandwidth:

$$
w_{u}=A_{0} \cdot w_{p 1}=\frac{g_{m 1}}{C_{c}}
$$

Because there is no DC current pass the NMOS tube M14, it works in the linear region, and it is equivalent of a resistor.

$$
R_{c}=\frac{1}{\mu_{n} C_{o x}\left(\frac{w}{L}\right)_{14} V_{\text {eff } 14}}
$$

The role of the M14 tube is to eliminate the right half zero which is introducted by the Miller capacitor Cc. Fig. 2 is the small signal model of frequency compensation for Fig. 1. 


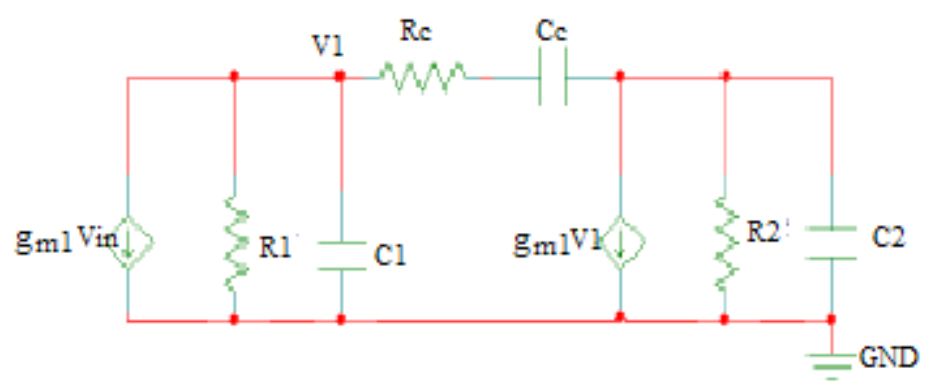

Fig.2 the small signal model of frequency compensation

Assuming Rc=0, using the node current analysis way, we can get:

$$
\begin{aligned}
& g_{m 1} V_{m 1}+V_{1} \cdot\left(\frac{1}{R_{1}}+s C_{1}\right)+\left(V_{1}-V_{\text {out }}\right) \cdot s C_{c}=0 \\
& g_{m 6} V_{1}+V_{\text {out }} \cdot\left(\frac{1}{R_{2}}+s C_{2}\right)+\left(V_{\text {out }}-V_{1}\right) \cdot s C_{c}=0
\end{aligned}
$$

The following equation can be obtained by the above equations combined solution:

In middle :

$$
\frac{V_{\text {out }}}{V_{\text {in }}}=\frac{g_{m 1} g_{m 6} R_{1} R_{2}\left(1-\frac{s C_{c}}{g_{m 6}}\right)}{a_{2} s^{2}+a_{1} s+1}
$$

$$
\begin{aligned}
& a_{2}=R_{1} R_{2}\left(C_{1} C_{2}+C_{1} C_{c}+C_{2} C_{c}\right) \\
& a_{1}=\left(C_{1}+C_{c}\right) R_{2}+g_{m 6} R_{1} R_{2} C_{c}
\end{aligned}
$$

In the actual circuit, the two poles are very far apart, $w_{p 2}>>w_{p 1}$

$$
H(s)=\left(1-\frac{s}{w_{p 1}}\right)\left(1-\frac{s}{w_{p 2}}\right)=1-\frac{s}{w_{p 1}}+\frac{s^{2}}{w_{p 1} w_{p 2}}
$$

So, $\frac{1}{C_{c}\left(\frac{1}{g_{m 6}}-R_{c}\right)}=\frac{-g_{m 6}}{C_{1}+C_{2}}$

The value of Rc is: $R_{c}=\frac{1}{g_{m 6}}\left(1+\frac{C_{1}+C_{2}}{C_{c}}\right)$

We can get

$$
\begin{array}{ll} 
& g_{m 6} C_{c}^{2}>g_{m 1} C_{1} C_{2}+g_{m 1} C_{1} C_{c}+g_{m 1} C_{1}^{2}>g_{m 1} C_{1} C_{2} \\
\text { And } \quad C_{c}>\sqrt{\frac{g_{m 1} C_{1} C_{2}}{g_{m 6}}}
\end{array}
$$

We use the Spice to simulation the Op, and it is based on standard 0.18um CMOS technology, with 3.3 power supply.

\section{Analysis of Result:}

The Fig 3 has showed that the amplifier DC gain is 106db, the gain-bandwidth is $167 \mathrm{MHZ}$, and the phase margin is $75^{\circ}$. Table1 has given the measured performance, the simulation showed the ADC can achieve 8bits resolution and 10Msamples/s. 


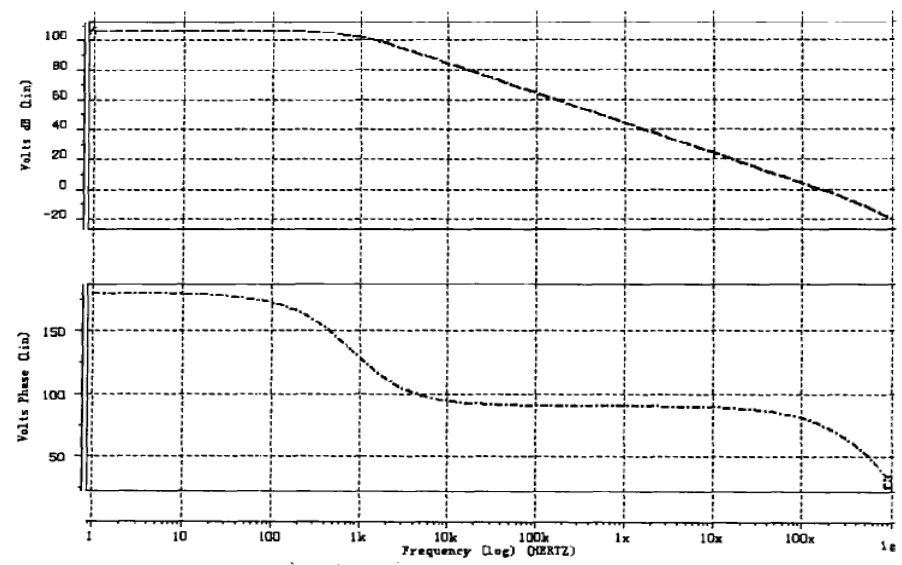

Fig.3 the performance of 8 Bits Pipeline ADC

Table 1 Measured Performance

\begin{tabular}{|l|l|}
\hline Supply voltage & $3.3 \mathrm{~V}$ \\
\hline Load capacitance & $3 \mathrm{pf}$ \\
\hline Common in-put voltage & $1.65 \mathrm{~V}$ \\
\hline Output Dynamic Range & {$[0.33 \mathrm{~V}, 2.97 \mathrm{~V}]$} \\
\hline Open-loop DC gain & $106 \mathrm{db}$ \\
\hline Phase margin & $75^{0}$ \\
\hline Conversion rate & $35 \mathrm{~V} / \mathrm{us}$ \\
\hline
\end{tabular}

\section{Conclusion}

Analog-to-digital conversion can be accomplished in numerous ways, but apart from flash converters, only pipeline of analog-to-digital conversion (ADC) are well known for producing accurate, medium speed conversion of analog signals. The 8 bit pipeline ADC was proposed for low-power low-cost CMOS integrated system. The experimental results showed that the proposed circuits achieves $10 \mathrm{Ms} / \mathrm{s}$ sample with power consumption at $3.3 \mathrm{~V}$. This device is suitable for standard CMOS technology VLSI implementation. It is well applied when embedded into system-on-chip (SoC) circuit designs.

\section{Acknowledgements}

This work was financially supported by the Chinese Natural Science Foundation (61306093 and 61401075).

\section{References}

[1] J. Mitola, Guest editorial on software radios, IEEE Communication, 1995, 33, (5), pp. 24-25

[2] W. H. W. Tuttlebee, Software radio technology: a european perspective, IEEE Communication, 1999, 37, (2), pp.118-123.

[3] T. Yoshida, M. Akagi, and M. Sasaki, A 1V supply successive approximation ADC with rail-to-rail input voltage range, IEEE Circuits and Systems, 2005, 1, (2):192-195.

[4] J. Li, X.Zeng, and J. Zhang: Design of an ADC for subsampling video applications, IEEE Analog Integrated Circuits and Signal Processing, 2006, 5, (9):303-312.

[5] Silvia, D., Davide, V., and Andrea, B. : 'A 6-bit 1.2 GHz interleaved SAR ADC in 90 nm CMOS' Research in Microelectronics and Electronics, 2006, 12, (15):301-304. 